\title{
On noncommutative geometry of the Standard Model: fermion multiplet as internal forms
}

\author{
Ludwik Dąbrowski
}

\begin{abstract}
We unveil the geometric nature of the multiplet of fundamental fermions in the Standard Model of fundamental particles as a noncommutative analogue of de Rham forms on the internal finite quantum space.
\end{abstract}

Mathematics Subject Classification (2010). Primary 58B34; Secondary 46L87, 81T13.

Keywords. spinors, differential forms, noncommutative geometry; spectral triples; Standard Model.

\section{Introduction}

From the conceptual point of view the Standard Model (S.M.) of fundamental particles and their interactions is a particular model of $U(1) \times S U(2) \times S U(3)$ gauge fields (bosons) minimally coupled to matter fields (fermions), plus a Higgs field (boson). After the second quantization with gauge fixing, spontaneous symmetry breaking mechanism, regularization and perturbative renormalization it extremely well concords with the experimental data. Even so (unreasonably) successful it however does not explain (though somewhat constrains) the list of particles, in particular the existence of 3 families, contains several parameters and does not include the fourth known interaction: gravitation, with its own fundamental symmetry: general relativity or diffeomorphisms. There have been various attempts to settle some of the above shortcomings: GUT based on a simple group $S U(5)$ or $S O(10)$, modern variants of old Kaluza-Klein model with 'compactified' internal dimensions, and others more recent and fashionable, that are still under extensive massive study.

Of our interest in this note is another distinct approach to the S.M. in the framework of noncommutative geometry by A. Connes et.al., see e.g. [6], which is not so widely known among physicists. It interprets the multiplet of fundamental fermions as a field on a finite quantum space, on which the would be "coordinates", as well as the algebra of "functions" fail to commute.

This work is part of the project Quantum Dynamics sponsored by EU-grant RISE 691246 and Polish Government grant 317281, and was partially supported by by the Polish Ministry of Science and Higher Education 2015-2019 matching fund 3542/H2020/2016/2. 
In this note we focus on its deeper geometric structure, aiming to shed more light on the nature of this internal quantum space. We shall review the key results of the two recent papers [7,8, explaining in more detail the classical (commutative) motivation behind them. For that some well known material in differential geometry will be presented from the viewpoint of the so called spectral triples; with the only new contribution in the last part of Subsection 2.2 regarding their KO-dimension.

\section{Introduction}

The noncommutative formulation $\nu$ S.M. of the Standard Model takes its cue from its geometry which in mathematical terminology corresponds to a connection (locally a multiplet of vector fields) whose structure group is $U(1) \times S U(2) \times S U(3)$ on (a multiplet) of spinors, together with a doublet of scalar fields. Although it does not renounce of groups, $\nu$ S.M. is however based primarily on algebras. Moreover to the 75 years-old Gelfand-Naimark (anti)equivalence:

$$
\text { topological spaces } \longleftrightarrow \text { commutative } C^{*} \text {-algebras }
$$

and to the Serre-Swan equivalence:

$$
\text { vector bundles } \longleftrightarrow \text { modules }
$$

it adjoins two other ingredients to encode such structures as smoothness, calculus and (Riemannian) metric on a space $M$. The first one is a Hilbert space $H$ that carries a unitary representation of a (possibly noncommutative) *-algebra $A$, and so obviously also of its norm completed $C^{*}$-algebra. The second one is an analogue of the Dirac operator on $H$. Together with a *algebra $A$ they satisfy certain analytic conditions: $D$ is selfadjoint, $[D, a]$ are bounded $\forall a \in A$ and $(D-z)^{-1}$ are compact for $z \in \mathbb{C} \backslash \mathbb{R}$, so that they form the so called spectral triple (S.T.) [5]

$$
(A, H, D) \text {. }
$$

Such a S.T. is even if there is a $\mathbb{Z}_{2}$-grading $\chi$ of $H, \chi^{2}=1, \chi^{\dagger}=\chi$, with which all $a \in A$ commute and $D$ anticommutes. Furthermore it is real if there is a $\mathbb{C}$-antilinear isometric operator $J$ on $H$, such that denoting $B^{\prime}$ the commutant of $B \subset \mathcal{B}(H)$,

$$
J A J^{-1} \subset A^{\prime},
$$

which is often termed order 0 condition. We say that a real S.T. satisfies the order 1 condition if

$$
J A J^{-1} \subset[D, A]^{\prime}
$$

and the order 2 condition 11 if

$$
J[D, A] J^{-1} \subset[D, A]^{\prime} .
$$

\footnotetext{
${ }^{1}$ while the order 1 condition means classically that $D$ is order 1 differential operator, order 0 and 2 conditions don't have such interpretation
} 
The $A$-bimodule spanned by $[D, A]$ is often called space of 1 -forms, and the algebra generated by $A$ and $[D, A]$ the space of all forms for the Dirac calculus (with the exterior derivative given by the commutator with $D$ ). Motivated by the classical examples (cf. the next subsections) slightly abusing the terminology we will call, quite as in [10, Cliford algebra the complex *-algebra $\mathcal{C} \ell_{D}(A)$ generated by $A$ and $[D, A]$.

Note that for noncommutative $A$ a priori there is no right action of $A$ on $H$, but given $J$ there is one:

$$
h a:=J a^{*} J^{-1} h,
$$

that commutes with the left action due to the order 0 condition and so $H$ becomes an $A$ - $A$ bimodule. Furthermore, if the order 1 condition holds $H$ becomes a $\mathcal{C} \ell_{D}(A)-A$ bimodule, and if the 2 nd order condition holds $H$ becomes even a $\mathcal{C} \ell_{D}(A)-\mathcal{C} \ell_{D}(A)$ bimodule.

Connes formulated few other important properties of real spectral triples. One of them requires that the following identities are satisfied

$$
\begin{gathered}
J^{2}=\epsilon \mathrm{id}, \\
D J=\epsilon^{\prime} J D,
\end{gathered}
$$

and in even case

$$
\chi J=\epsilon^{\prime \prime} J \chi,
$$

where the three signs $\epsilon, \epsilon^{\prime}, \epsilon^{\prime \prime} \in\{+,-\}$ specify the so called KO-dimension modulo 8:

\begin{tabular}{|c|c|c|c|c|c|c|c|c|}
\hline$n$ & 0 & 1 & 2 & 3 & 4 & 5 & 6 & 7 \\
\hline$\epsilon$ & + & + & - & - & - & - & + & + \\
\hline$\epsilon^{\prime}$ & + & - & + & + & + & - & + & + \\
\hline$\epsilon^{\prime \prime}$ & + & & - & & + & & - & \\
\hline
\end{tabular}

(If dimension $n$ is even one can alternatively use $\chi J$ as a new real structure, which changes the parameter $\epsilon^{\prime}$ to $-\epsilon^{\prime}$, and $\epsilon$ to $\left.\epsilon \epsilon^{\prime \prime}\right)$.

\subsection{Canonical spectral triple}

A prototype example is the canonical S.T. on a closed oriented spin manifold $M$ of dimension $n$ equipped with a Riemannian metric $g$ :

$$
\left(C^{\infty}(M), L^{2}(S), \not D\right)
$$

Here $C^{\infty}(M)$ is the algebra of smooth complex functions on $M, S$ is the $\operatorname{rank}_{\mathbb{C}}=2^{[n / 2]}$ bundle of Dirac spinors on $M$, whose sections carry a faithful irreducible representation

$$
\gamma: \Gamma(\mathcal{C} \ell(M)) \stackrel{\approx}{\longrightarrow} \operatorname{End}_{C^{\infty}(M)} \Gamma(S) \approx \Gamma(S) \otimes_{C^{\infty}(M)} \Gamma(S)^{*},
$$

of the algebra of sections of the (simple part of) complex Clifford bundle $\mathcal{C} \ell(M)$, generated by $v \in T M$ with relation $v^{2}+g(v, v)=0$. Furthermore $\not D$ is the usual Dirac operator

$$
\not D=\gamma \circ \tilde{\nabla}=\sum_{j}^{n} \gamma\left(e_{j}\right) \tilde{\nabla}_{e_{j}},
$$


where $\tilde{\nabla}$ is a lift to $S$ of the Levi-Civita connection on $M$ and $e_{j}, j=1, \ldots, n$, is a local oriented orthonormal basis of $T M$.

One has

$$
[\not D, f]=\gamma(\mathrm{d} f), \quad f \in C^{\infty}(M),
$$

or, what is the same, the symbol of $\not D$ is

$$
\sigma_{\not D}(\xi)=-i \gamma(\xi), \quad \xi \in T^{*} M
$$

where we have identified $T M \approx T^{*} M$, and so $\nabla f$ with $d f$, using the metric $g$. Note that the operators of that form together with functions generate an isomorphic copy of the Clifford algebra $\Gamma(\mathcal{C} \ell(M))$.

If $\operatorname{dim} M$ is even there is also a $\mathbb{Z}_{2}$-grading $\chi_{S}$ of $L^{2}(S)$, with which all $a \in A$ commute and $\not D$ anticommutes. It should be mentioned that $\not D$ is an elliptic operator and its index, or more precisely the Fredholm index of $\left.\not D\right|_{\Gamma(S)}: \Gamma(S) \rightarrow \Gamma(S)$, where $\Gamma\left(S^{ \pm}\right)$are \pm 1 eigenspaces of $\chi_{S}$, plays an important role in geometry and applications to physics. It can be expressed in terms of the characteristic class called $\hat{A}$-genus, a topological invariant of $M$.

Furthermore there is a real structure (known as charge conjugation) $J_{S}$ on $L^{2}(S)$, that satisfies the order 0 condition (1) and the order 1 condition (2), but not the order 2 condition (3). Indeed, $J_{S}$ and (8) obey a stronger version of (11) and (2) which excludes (3). Namely the norm closure of $C^{\infty}(M)$, that is the algebra $C(M)$ of continuous functions on $M$, is the maximal commutant in $B\left(L^{2}(S)\right)$ of the norm closure of $\mathcal{C} \ell_{\not D}\left(C^{\infty}(M)\right)=C^{\infty}(M)\left[\not D, C^{\infty}(M)\right]$, which is just the algebra of continuous sections $\Gamma(\mathcal{C} \ell(M))$ of the (complexified) Clifford bundle $\mathcal{C} \ell(M)$ on $M$ in the Dirac representation. We can thus say that "the Dirac spinor fields provide a Morita equivalence $C(M)$ $\Gamma(\mathcal{C} \ell(M))$ bimodule".

As a matter of fact for the latter property it suffices that $M$ is $\operatorname{spin}_{c}$, that can be defined by any of the following three equivalent statements:

i) there exist a principal $\operatorname{Spin}_{c}(n)$-bundle, such that the vector bundle associated with the representation $\rho \times \square$ is isomorphic to the tangent bundle $T(M)$;

ii) $S O(n)$-bundle of oriented orthogonal frames lifts to $\operatorname{Spin}_{c}(n)$;

iii) the second Stiefel-Whitney class $w_{2}(M)$ is a modulo 2 reduction of a class in $H^{2}(M, \mathbb{Z})$.

Here $\operatorname{Spin}_{c}(n)$ is the quotient group of $\operatorname{Spin}(n) \times U(1)$ by the subgroup $\mathbb{Z}_{2}^{\text {diag }}=\{(1,1),(-1,-1)\}, \rho: \operatorname{Spin}(n) \rightarrow S O(n)$ is the nontrivial double covering and $\square: U(1) \rightarrow U(1)$ is the square map.

Importantly however the property that an oriented Riemannian manifold $M$ is $\operatorname{spin}_{c}$ is actually tantamount 12 to

$$
\exists \text { a Morita equivalence } \mathcal{C} \ell(M)-C(M) \text { bimodule } \Sigma \text {. }
$$

Indeed when (11) holds then automatically $\Sigma \approx \Gamma(S)$, where $S$ is the $\mathbb{C}$-vector bundle of Dirac spinors on $M$. 
Therefore $\operatorname{spin}_{c}$ manifolds lend itself to noncommutative generalization via the algebraic property (11) by taking advantage of the definition of Clifford algebra $\mathcal{C} \ell_{D}(A)$. Next, the algebraic characterization of spin manifolds also admits a noncommutative generalization as the condition (11) plus a real structure (charge conjugation) $J$ that implements it.

We remark that the canonical S.T. fully encodes the geometric data on $M$, that can be indeed reconstructed 4 from a commutative S.T. with certain few additional properties. One of these properties requires that KOdimension, defined by (4), (5), (6), is equal for the operators $\not D, \chi_{S}, J_{S}$ to the dimension of $M$ modulo 8 .

\subsection{Hodge-de Rham spectral triple}

The canonical S.T. (8) is not the only natural S.T. On any oriented closed Riemannian manifold $M$ there is also

$$
\left(C^{\infty}(M), L^{2}(\Omega(M)), d+d^{*}\right),
$$

where $\Omega(M)$ is the graded space of complex de Rham differential forms on $M, d$ is the exterior differential and $d^{*}$ is its adjoint.

The operator $d+d^{*}$ is actually Dirac-type since

$$
d+d^{*}=\lambda \circ \nabla
$$

where

$$
\left.\lambda: \Gamma(\mathcal{C} \ell(M)) \rightarrow \operatorname{End}_{C \infty}(M) \Omega(M), \lambda(v)=v \wedge-v\right\lrcorner, v \in T^{*} M \approx T M,
$$

is the (reducible) faithful complex representation on $\Omega(M)$ of the algebra of sections $\Gamma(\mathcal{C} \ell(M))$ of the (complexified) Clifford bundle $\mathcal{C} \ell(M)$ over $M$. The formula (13) means that

$$
\left[d+d^{*}, f\right]=\lambda(\mathrm{d} f), \quad f \in C^{\infty}(M),
$$

what is also the same as the symbol of $d+d^{*}$ being

$$
\sigma_{d+d^{*}}(\xi)=-i \lambda(\xi), \quad \xi \in T^{*} M .
$$

Note that as for the canonical $\not D$ the operators of the form (13) together with functions generate $\Gamma(\mathcal{C} \ell(M))$, and thus indeed $\mathcal{C} \ell_{d+d^{*}}\left(C^{\infty}(M)\right) \approx \Gamma(\mathcal{C} \ell(M))$.

The representation $\lambda$ is equivalent to the left regular self-representation of $\Gamma(\mathcal{C} \ell(M))$, via the isomorphism of vector bundles $\mathcal{C} \ell(M) \approx \Omega(M)$. There is also an anti-representation

$$
\left.\rho: \Gamma(\mathcal{C} \ell(M)) \rightarrow \operatorname{End}_{C}^{\infty}(M) \Omega(M), \lambda(v)=(v \wedge+v\lrcorner\right) \chi_{\Omega}, v \in T^{*} M \approx T M,
$$

where

$$
\chi_{\Omega}= \pm 1
$$

on even forms $\Omega(M)^{\text {even }}$, respectively odd forms $\Omega(M)^{\text {odd }}$. It is equivalent to the right regular self-antirepresentation of $\Gamma(\mathcal{C} \ell(M))$.

Furthermore, since the endomorphisms $\lambda(v)$ and $\rho\left(v^{\prime}\right)$ commute, $\Omega(M)$ is a $\Gamma(\mathcal{C} \ell(M))-\Gamma(\mathcal{C} \ell(M))$ bimodule, which is equivalent to $\Gamma(\mathcal{C} \ell(M))$. Thus, $\Omega(M)$ is actually a self-Morita equivalence $\Gamma(\mathcal{C} \ell(M))-\Gamma(\mathcal{C} \ell(M))$ bimodule, 
the property which in fact provides its unambiguous characterization up to a tensor product with sections of a complex line bundle.

The operator $\chi_{\Omega}$ (17) always defines a $\mathbb{Z}_{2}$-grading of $L^{2}(\Omega(M))$ according to the parity of forms. If $\operatorname{dim} M=n=2 m$ (even) there is also another grading $\chi_{\Omega}^{\prime}$ given by the normalized Hodge star operator, defined in terms of a local orthonormal oriented basis $e^{j}, j=1, \ldots, n, n=2 m$ of $T^{*} M$ by

$$
\chi_{\Omega}^{\prime}\left(e^{j_{1}} \wedge \cdots \wedge e^{j_{k}}\right)=i^{k(k-1)+m} e^{j_{k+1}} \wedge \cdots \wedge e^{j_{n}}, \quad 0 \leq k \leq n,
$$

where $j_{1}, \ldots j_{n}$ is an even permutation of $1, \ldots n$.

Both the gradings $\chi_{\Omega}$ and $\chi_{\Omega}^{\prime}$ commute with $a \in C^{\infty}(M)$ and anticommute with $d+d^{*}$. As well known, they play important role for the index of the elliptic operator $d+d^{*}$. More precisely the Fredholm index of

$$
\left.\left(d+d^{*}\right)\right|_{\Omega(M)^{\text {even }}: \Omega(M)^{\text {even }} \rightarrow \Omega(M)^{\text {odd }}}
$$

computes the Euler character of $M$, while the index of

$$
\left.\left(d+d^{*}\right)\right|_{\Omega(M)^{s}}: \Omega(M)^{s} \rightarrow \Omega(M)^{a},
$$

where $\Omega(M)^{s}$ and $\Omega(M)^{a}$ are respectively the \pm 1 eigenspaces of $\chi_{\Omega}^{\prime}$, computes the signature of $M$.

Furthermore there is also a real structure $J_{\Omega}$ on $L^{2}(\Omega(M))$ given just by the complex conjugation of forms. It satisfies the conditions (11) and (2) but definitely not (3) and therefore can not implement the $\Gamma(\mathcal{C} \ell(M))-\Gamma(\mathcal{C} \ell(M))$ self-Morita equivalence as above.

In order to implement this equivalence, we need another real structure $J_{\Omega}^{\prime}$ on $\Omega(M)$, that interchanges the actions $\lambda$ and $\rho$. It turns out that it can be defined as

$$
J_{\Omega}^{\prime}\left(e^{j_{1}} \wedge \cdots \wedge e^{j_{k}}\right)=e^{j_{k}} \wedge \cdots \wedge e j_{1}, \quad 0 \leq k \leq n,
$$

which corresponds to the main anti-involution on $\Gamma(\mathcal{C} \ell(M))$ and can be simply written on $\Omega^{k}(M)$ as

$$
J_{\Omega}^{\prime}=(-)^{k(k-1) / 2} \circ \text { c.c. }
$$

This real structure satisfies all the order conditions (11), (2) and (3) and does implement the $\Gamma(\mathcal{C} \ell(M))-\Gamma(\mathcal{C} \ell(M))$ self-Morita equivalence as above.

We mention that for the operators $d+d^{*}, \chi_{\Omega}, J_{\Omega}$ one gets the signs $\epsilon=1, \epsilon^{\prime}=1, \epsilon^{\prime \prime}=1$ and so the KO-dimension is 0 . Instead for the operators $d+d^{*}, \chi_{\Omega}^{\prime}, J_{\Omega}$ the signs are $\epsilon=1, \epsilon^{\prime}=1, \epsilon^{\prime \prime}=(-1)^{m}$ and so the KO-dimension is 0 if $\mathrm{n}=0 \bmod 4$, and 6 if $\mathrm{n}=2 \bmod 4$ [A. Rubin, MSc Thesis].

As far as the operators $d+d^{*}, \chi_{\Omega}, J_{\Omega}^{\prime}$ are concerned we get the signs $\epsilon=1, \epsilon^{\prime}=1, \epsilon^{\prime \prime}=1$ and so the KO-dimension is 0 . Instead for the operators $d+d^{*}, \chi_{\Omega}^{\prime}, J_{\Omega}^{\prime}$ we get the signs $\epsilon=1$ and $\epsilon^{\prime}=1$, while on $\Omega^{k}(M)$ we obtain

$$
J_{\Omega}^{\prime} \chi_{\Omega}^{\prime}=(-1)^{k} \chi_{\Omega}^{\prime} J_{\Omega}^{\prime}
$$

in which the sign appears that depends on $k$. Thus in that case there is no overall sign $\epsilon^{\prime \prime}$ but in fact a grading given by $\chi_{\Omega}$. This feature generalizes somewhat the notion of KO-dimension which as known was tailored for the canonical spectral triple. 
Closing this section we remark that it is not clear whether, and with which additional conditions, this S.T. equiped with any combination of the gradings and real structures as above may faithfully encode the geometric data on $M$, that can be then reconstructed.

\section{Noncommutative formulation of the Standard Model: $\nu$ S.M.}

Concerning the underlying arena of $\nu$ S.M., see e.g. [6], it is

$(\operatorname{ordinary}(\operatorname{spin})$ manifold $M) \times($ finite quantum space $F)$, described by the algebra $C^{\infty}(M) \otimes A_{F} \approx C^{\infty}\left(M, A_{F}\right)$, where

$$
A_{F}=\mathbb{C} \oplus \mathbb{H} \oplus M_{3}(\mathbb{C}) .
$$

Here $\mathbb{H}$ is the (real) algebra of matrices of the form

$$
\left[\begin{array}{cc}
\alpha & \beta \\
-\bar{\beta} & \bar{\alpha}
\end{array}\right], \quad \alpha, \beta \in \mathbb{C},
$$

which is isomorphic to the algebra of quaternions.

The Hilbert space is

$$
L^{2}(S) \otimes H_{F},
$$

where

$$
H_{F}=\mathbb{C}^{96}=: H_{f} \otimes \mathbb{C}^{3},
$$

with $\mathbb{C}^{3}$ corresponding to 3 generations, and

$$
H_{f}=\mathbb{C}^{32} \simeq M_{8 \times 4}(\mathbb{C}) .
$$

The orthonormal basis of $H_{f}$ is labelled by particles and antiparticles, that we arrange as a $8 \times 4$ matrix

$$
\left[\begin{array}{cccc}
\nu_{R} & u_{R}^{1} & u_{R}^{2} & u_{R}^{3} \\
e_{R} & d_{R}^{1} & d_{R}^{2} & d_{R}^{3} \\
\nu_{L} & u_{L}^{1} & u_{L}^{2} & u_{L}^{3} \\
e_{L} & d_{L}^{1} & d_{L}^{2} & d_{L}^{3} \\
\bar{\nu}_{R} & \bar{e}_{R} & \bar{\nu}_{L} & \bar{e}_{L} \\
\bar{u}_{R}^{1} & \bar{d}_{R}^{1} & \bar{u}_{L}^{1} & \bar{d}_{L}^{1} \\
\bar{u}_{R}^{2} & \bar{d}_{R}^{2} & \bar{u}_{L}^{2} & \bar{d}_{L}^{2} \\
\bar{u}_{R}^{3} & \bar{d}_{R}^{3} & \bar{u}_{L}^{3} & \bar{d}_{L}^{3}
\end{array}\right]
$$

where the indices 1,2,3 correspond to the color quantum number. 
The representation $\pi_{F}$ of $A_{F}$ is diagonal in generations and $\pi_{F}(\lambda, q, m)$ is given on $H_{f}$ i by left multiplication by the matrix:

$$
\left[\begin{array}{c}
{\left[\begin{array}{ll|ll}
\lambda & 0 & 0 & 0 \\
0 & \bar{\lambda} & 0 & 0 \\
\hline 0 & 0 & & \\
0 & 0 & & q
\end{array}\right]} \\
\\
\end{array}\right.
$$

Note that $\pi_{F}\left(A_{F}\right)$ is a real $*$-algebra of operators, and to get its complexification $\mathbb{A}_{F}$ just replace $\bar{\lambda}$ by an independent $\lambda^{\prime} \in \mathbb{C}$, and take $q \in M_{2}(\mathbb{C})$.

The grading (the chirality operator) is

$$
\chi_{M} \otimes \chi_{F},
$$

where $\chi_{F}$ is diagonal in generations and on $H_{f}$ reads:

$$
\chi_{F}=\left[\begin{array}{lll}
1_{2} & & \\
& -1_{2} & \\
& & 0_{4}
\end{array}\right] \otimes 1_{4}+\left[\begin{array}{ll}
0_{4} & \\
& -1_{4}
\end{array}\right] \otimes\left[\begin{array}{ll}
1_{2} & \\
& -1_{2}
\end{array}\right] .
$$

The real conjugation is

$$
J_{M} \otimes J_{F}
$$

where $J_{F}$ is also diagonal in generations and on $H_{f}$ reads:

$$
J_{F}\left[\begin{array}{l}
v_{1} \\
v_{2}
\end{array}\right]=\left[\begin{array}{l}
v_{2}^{*} \\
v_{1}^{*}
\end{array}\right]
$$

that satisfies $J_{F}^{2}=1$, the order 0 condition:

$$
\left[a, J_{F} b J_{F}^{-1}\right]=0 \quad \forall a, b \in A_{F},
$$

and the order 1 condition:

$$
\left[[D, a], J_{F} b J_{F}^{-1}\right]=0 \quad \forall a, b \in A_{F}
$$

(as in the classical case).

Finally, the Dirac operator is

$$
D=\not D_{M} \otimes \mathrm{id}+\chi_{M} \otimes D_{F},
$$


where $D_{F}$ employed by Chamseddine-Connes' reads on $H_{F}$

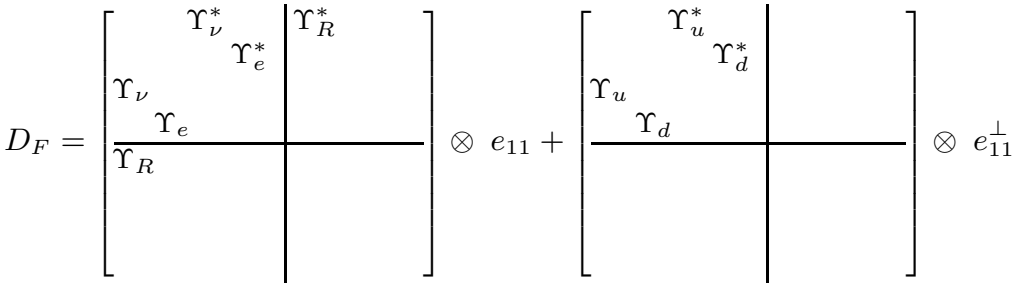

$$
\begin{aligned}
& +e_{55} \otimes\left[\begin{array}{cc|cc}
0 & 0 & \Upsilon_{\nu}^{*} & 0 \\
0 & 0 & 0 & \Upsilon_{e}^{*} \\
\hline \Upsilon_{\nu} & 0 & 0 & 0 \\
0 & \Upsilon_{e} & 0 & 0
\end{array}\right]+\left(e_{66}+e_{77}+e_{88}\right) \otimes\left[\begin{array}{cc|cc}
0 & 0 & \Upsilon_{u}^{*} & 0 \\
0 & 0 & 0 & \Upsilon_{d}^{*} \\
\hline \Upsilon_{u} & 0 & 0 & 0 \\
0 & \Upsilon_{d} & 0 & 0
\end{array}\right] \text {. }
\end{aligned}
$$

Here the first tensor factor acts by the left matrix multiplication and the second one by the right matrix multiplication, $e_{j k}$ are the usual matrix units, the empty spaces stand for 0 , and $\Upsilon$ 's are in $\operatorname{Mat}(N, \mathbb{C})$ with $N$ equal to the number of generations ( $N=3$ on the current experimental basis).

Concerning the data $D_{F}, \chi_{F}$ and $J_{F}$ given above the KO-dimension comes as 6 .

\subsection{Properties of $\nu$ S.M.}

With the ingredients as listed in the previous section one gets:

- the group $\mathcal{G}:=\left\{U=u J_{F} u J_{F}^{-1} \mid u \in A_{F}\right.$, det $\left.U=1\right\}$ turns out to be isomorphic (up to a finite center) with the gauge group $U(1) \times S U(2) \times$ $S U(3)$ of the S.M. (also as functions on $M$ );

- all the fundamental fermions in $H$ have the correct S.M. charges with respect to $\mathcal{G}$ broken to $U(1)_{e m} \times S U(3)$;

- the 1 -forms $a\left[D_{F}, b\right], a, b \in C^{\infty}\left(M, A_{F}\right)$ yield the gauge fields $A_{\mu}, W^{ \pm}$, $Z, G_{\mu}$ of the S.M. (from the part $D_{M}$ of $D$ ), plus the weak doublet complex scalar Higgs field (from the part $D_{F}$ of $D$ ).

The merits of the noncommutative formulation are:

- it treats discrete and continuous spaces (or variables) on the same footing;

- both the gauge and the Higgs field arise as parts of a connection;

- the appearance of solely fundamental representations of $\mathcal{G}$ in the S.M. gets an explanation as the fact that they are the only irreducible representations of simple algebras;

- there is an elegant spectral action $\operatorname{Tr} f(D / \Lambda)$, that reproduces the bosonic part of $\mathcal{L}_{S . M}$. as the lowest terms of asymptotic expansion in $\Lambda$, and the matter action $\langle\phi, D \phi\rangle$ for the (Wick-rotated) fermionic part;

- it couples in a natural way to gravity on $M$;

- is claimed [2,3, to predict new relations among the parameters of S.M.

Some of the shortcomings still present are as in the usual S.M.:

the 3 generations (families) put by hand, several free parameters, though 
most of them incorporated into a single geometric quantity: $D_{F}$. Others are the unimodularity condition to be posed on the gauge group $\mathcal{G}$ and a special treatment needed for the two kinds of fermion doublings due to the presence of chirality \pm 1 and particles/antiparticles both in $L^{2}(S)$ and $H_{F}$.

\subsection{The geometric nature of $H_{f}$}

The above "almost commutative" geometry is described by a S.T.

$$
\left(C^{\infty}(M), L^{2}(S), \not D\right) \times\left(A_{F}, H_{f}, D_{F}\right),
$$

that is mathematically a product of the "external" canonical S.T. on spin manifold $M$ with the "internal" finite S.T.

Few quite natural questions are in order concerning the geometric interpretation of the internal S.T. $\left(A_{F}, H_{f}, D_{F}\right)$ :

Does it also correspond to a (noncommutative) spin manifold?

Are the elements of $H_{f}$ "spinors" in some sense?

In particular "Dirac spinors"?

Or does it correspond rather to de-Rham forms?

Or else?

To answer these questions, motivated by the classical case as in Sect.2.1 the following definition has been formulated for a general unital S.T. :

Definition (7]). A real spectral triple $(A, H, D, J)$ is called spin (and elements of $H$ are quantum Dirac spinors) if $H$ is a Morita equivalence $\mathcal{C} \ell_{D}(A)-J A J^{-1}$ bimodule (i.e. after norm-completion the algebras $\mathcal{C} \ell_{D}(A)$ and $J A J^{-1}$ are maximal one with respect to the other).

Is then the internal S.T. of $\nu$ S.M. spin, like the external one that is given by the canonical S.T. on $M$ ?

Building on and extending the classifications of [9] and [1] the answer in [7] is negative. In fact therein after a systematic search an element

$$
X=e_{55} \otimes\left(1-e_{11}\right)
$$

has been found, such that $X \in \mathcal{C} \ell_{D}(A)^{\prime}$ but $X \notin J A J$. A possible way to circumvent this "no go" has been suggested by employing a different grading and adding two extra non-zero matrix elements of $D_{F}$, the status of which however requires a further scrutiny (since though desirable for the correct renormalized Higgs mass, they would have unobserved couplings to fermions).

But then, without such additions, may be the internal S.T. of $\nu$ S.M. is rather an analogue of the other natural classical spectral triple, namely de-Rham forms?

To answer this question we have to formulate also these notions noncommutatively using the algebraic description of the Hodge-de Rham spectral triple with the grading $\chi_{\Omega}$ and real structure $J_{\Omega}^{\prime}$ as in Section 2.2

Definition (cf. [8]). A spectral triple $(A, H, D)$ is called complex Hodge (and vectors in $H$ complex quantum de-Rham forms) if $H$ is a Morita equivalence $\mathcal{C} \ell_{D}(A)-\mathcal{C} \ell_{D}(A)$ bimodule (i.e. after norm-completion these algebras are maximal one with respect to the other). 
A complex Hodge spectral triple $(A, H, D)$ with real structure $J$ is called

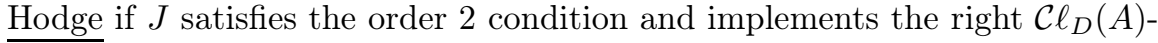
action.

The following theorem provides the answer in the case of one generation and thus for $\Upsilon^{\prime} s \in \mathbb{C}$ in (26).

Theorem ([8]). For the internal spectral triple of the $\nu$ S.M. with one generation the Hodge property holds whenever $\Upsilon_{x} \neq 0, \forall x \in\{\nu, e, u, d\}$ and

$$
\left|\Upsilon_{\nu}\right| \neq\left|\Upsilon_{u}\right| \quad \text { or } \quad\left|\Upsilon_{e}\right| \neq\left|\Upsilon_{d}\right| \text {. }
$$

In the rest of this section we will sketch the steps of the proof.

First by direct computation we find that the commutant of $A_{F}$ in $M_{8}(\mathbb{C})$ is the algebra $C_{F}$ with elements of the form

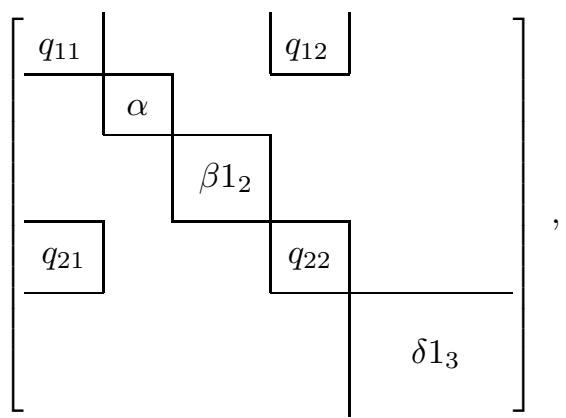

where $\alpha, \beta, \delta \in \mathbb{C}, q=\left(q_{i j}\right) \in M_{2}(\mathbb{C})$. Consequently the commutant of $A_{F}$ in $\operatorname{End}_{\mathbb{C}}(H)$ is $A_{F}^{\prime}=C_{F} \otimes M_{4}(\mathbb{C}) \simeq M_{4}(\mathbb{C})^{\oplus 3} \oplus M_{8}(\mathbb{C})$ of complex dimension 112.

Next $J_{F} A_{F} J_{F} \subset \operatorname{End}_{\mathbb{C}}\left(H_{F}\right)$ consists of elements of the form:

$$
\left[\begin{array}{ll}
1_{4} & 0_{4} \\
0_{4} & 0_{4}
\end{array}\right] \otimes\left[\begin{array}{c|ccc}
\lambda & 0 & 0 & 0 \\
\hline 0 & & \\
0 & & m \\
0 & &
\end{array}\right]+\left[\begin{array}{ll}
0_{4} & 0_{4} \\
0_{4} & 1_{4}
\end{array}\right] \otimes\left[\begin{array}{cc|cc}
\lambda & 0 & 0 & 0 \\
0 & \bar{\lambda} & 0 & 0 \\
\hline 0 & 0 & \\
0 & 0 & q
\end{array}\right],
$$

where the first factors of the tensor product acts by left matrix multiplication and the second factor by the right matrix multiplication.

Note that $A$ and $A_{\mathbb{C}}$ have the same commutant in $\operatorname{End}_{\mathbb{C}}\left(H_{F}\right)$. The map $a \mapsto J_{F} \bar{a} J_{F}$ gives an isomorphism $A_{F} \rightarrow J_{F} A_{F} J_{F}$ and of their complexifications, and also the map $x \mapsto J_{F} \bar{x} J_{F}$ is an isomorphism between $A_{F}^{\prime}$ and $\left(J_{F} A_{F} J_{F}\right)^{\prime}$.

Therefore the commutant $\left(J_{F} A_{F} J_{F}\right)^{\prime}$ of $J_{F} A_{F} J_{F}$ has elements

$$
a \otimes e_{11}+\left[\begin{array}{ll}
b & \\
& c
\end{array}\right] \otimes e_{22}+\left[\begin{array}{ll}
b & \\
& d
\end{array}\right] \otimes\left(e_{33}+e_{44}\right)
$$

with $a \in M_{8}(\mathbb{C}), b, c, d \in M_{4}(\mathbb{C})$.

Furthermore $A_{F}^{\prime} \cap\left(J_{F} A_{F} J_{F}\right)^{\prime} \simeq \mathbb{C}^{\oplus 10} \oplus M_{2}(\mathbb{C})$. It follows that the complex dimension of $A_{F}^{\prime}+\left(J_{F} A_{F} J_{F}\right)^{\prime}$ is $2 \cdot 112-14=210$. The (real) subspace of hermitian matrices has real dimenson 210. 
Now we recall that any unital complex $*$-subalgebra of $\operatorname{End}_{\mathbb{C}}(H)$, where $\operatorname{dim} H<\infty$, is a finite direct sum of matrix algebras: $B \simeq \bigoplus_{i=1}^{s} M_{m_{i}}(\mathbb{C})$. The units $P_{i}, 1 \leq i \leq s$ of $M_{m_{i}}(\mathbb{C})$ are orthogonal projections and $H$ decomposes as $H \simeq \bigoplus_{i=1}^{s} H_{i}$, with

$$
H_{i}=P_{i} \cdot H \simeq \mathbb{C}^{m_{i}} \otimes \mathbb{C}^{k_{i}},
$$

where $k_{i}$ is multiplicity of the (unique) irreducible representation $\mathbb{C}^{m_{i}}$ of $M_{m_{i}}(\mathbb{C})$ in $H_{i}$, and $M_{m_{i}}(\mathbb{C})$ acts on the 1st factor of $\mathbb{C}^{m_{i}} \otimes \mathbb{C}^{k_{i}}$ by matrix product. Then one has the following lemma:

Lemma (A). The commutant of $B$ in $\operatorname{End}_{\mathbb{C}}(H)$ is $B^{\prime} \simeq \bigoplus_{i=1}^{s} M_{k_{i}}(\mathbb{C})$ and the action of $B^{\prime}$ on $H_{i} \simeq \mathbb{C}^{m_{i}} \otimes \mathbb{C}^{k_{i}}$ is given by matrix multiplication by $M_{k_{i}}(\mathbb{C})$ of the second factor in the tensor product.

We will also need:

Lemma (B). Let $(A, H, D, J)$ be a finite-dimensional real spectral triple. Assume that $B \subseteq \operatorname{End}_{\mathbb{C}}(H)$ is a unital complex $*$-algebra satisfying:

$$
\mathcal{C} \ell_{D}(A) \subseteq B \quad \text { and } \quad B^{\prime}=J B J^{-1} .
$$

The following are equivalent:

(a) $\mathcal{C} \ell_{D}(A)^{\prime}=J \mathcal{C} \ell_{D}(A) J^{-1}$ (the Hodge property)

(b) $\mathcal{C} \ell_{D}(A)^{\prime} \subseteq J B J^{-1}$

(c) $\mathcal{C} \ell_{D}(A)=B$.

Proof of Lemma (B).

$(\mathrm{a} \Rightarrow \mathrm{b})$ The hypothesis $\mathcal{C} \ell_{D}(A) \subseteq B$ implies $J \mathcal{C} \ell_{D}(A) J^{-1} \subseteq J B J^{-1}$; and thus from (a) follows (b).

$(\mathrm{b} \Rightarrow \mathrm{c}) \mathcal{C} \ell_{D}(A)^{\prime} \subseteq J B J^{-1}=B^{\prime}$ implies $B \subseteq \mathcal{C} \ell_{D}(A)$, and so using our assumptions: $\mathcal{C} \ell_{D}(A)=B$.

$(\mathrm{c} \Rightarrow \mathrm{a})$ If (c) holds then $B^{\prime}=J B J^{-1}$ translates to $\mathcal{C} \ell_{D}(A)^{\prime}=J \mathcal{C} \ell_{D}(A) J^{-1}$.

Now, proceeding with the proof of Theorem, we take

$$
B:=\mathbb{C} \oplus M_{3}(\mathbb{C}) \oplus M_{4}(\mathbb{C}) \oplus M_{4}(\mathbb{C})
$$

with $(\lambda, m, a, b) \in B$ represented on $H_{F}$ as

$$
\left[\begin{array}{cc}
\lambda & 0 \\
0 & m
\end{array}\right] \otimes e_{22} \otimes 1+a \otimes e_{11} \otimes e_{11}+b \otimes e_{11} \otimes\left(1-e_{11}\right) .
$$

Next we:

- check that $\mathcal{C} \ell_{D_{F}}\left(A_{F}\right) \subset B$;

- check that $B$ and $J_{F} B J_{F}^{-1}$ commute, and so $J_{F} B J_{F}^{-1} \subseteq B^{\prime}$;

- note that (32) is equivalent to the representation of $B$ on:

$$
\left(\mathbb{C} \otimes \mathbb{C}^{4}\right) \oplus\left(\mathbb{C}^{3} \otimes \mathbb{C}^{4}\right) \oplus\left(\mathbb{C}^{4} \otimes \mathbb{C}\right) \oplus\left(\mathbb{C}^{4} \otimes \mathbb{C}^{3}\right)
$$

given by matrix multiplication on the first factors; 
- use Lemma (A) to infer that

$$
B^{\prime} \simeq M_{4}(\mathbb{C}) \oplus M_{4}(\mathbb{C}) \oplus \mathbb{C} \oplus M_{3}(\mathbb{C}) \simeq B
$$

and so we have $J_{F} B J_{F}^{-1}=B^{\prime}$;

- find that $\mathcal{C} \ell_{D_{F}}\left(A_{F}\right)^{\prime} \subseteq J_{F} B J_{F}^{-1}$;

- finish the proof by Lemma (B).

\section{Conclusions}

The Connes-Chamseddine noncommutative formulation of the Standard Model interprets the geometry of the S.M. as gravity on the product of a spin manifold $M$ with a finite noncommutative 'internal' space $F$. The multiplet of fundamental fermions (each one a Dirac spinor on $M$ ) defines fields on $F$ that constitute $H_{F}$.

We show that the geometric nature of the latter one is not a noncommutative analogue of Dirac spinors on $F$ (unless $>2$ new parameters are introduced in the matrix $D_{F}$, so fields on $M$ with physical status under scrutiny) but rather of de-Rham forms on $F$ if the conditions (27) are satisfied (for one generation). Conversely, the geometric qualification of the internal spectral triple as being Hodge constrains somewhat the parameters $\Upsilon$ occurring in the matrix $D_{F}$.

What happens for 3 generations of particles and so $96 \times 96$ matrices?

It can be seen (not easily) that then the spin property also does NOT hold, and that as adverted in [1, indeed the order 2 condition $\mathcal{C} \ell_{D}(A)^{\prime} \supset J \mathcal{C} \ell_{D}(A) J$ holds. Whether the Hodge property is satisfied, or $H_{F}$ corresponds rather to three copies of quantum de Rham forms on $F$, is currently under investigation.

\section{References}

1. L. Boyle and S. Farnsworth, Non-Commutative Geometry, Non-Associative Geometry and the Standard Model of Particle Physics, New J. Phys. 16 (2014) 123027.

2. A.H. Chamseddine and A. Connes, Space-Time from the spectral point of view, in the Proceedings of the 12th Marcel Grossmann meeting, World Sci.Singapore, 2012.

3. A.H. Chamseddine and A. Connes, Resilience of the Spectral Standard Model, JHEP 1209 (2012) 104.

4. A. Connes, On the spectral characterization of manifolds, J. Noncommut. Geom. 7 (2013) 1-82.

5. A. Connes, Gravity coupled with matter and foundation of non-commutative geometry, Commun. Math. Phys. 182 (1996) 155-176.

6. A. Connes and M. Marcolli, Noncommutative geometry, quantum fields and motives, Colloquium Publications, vol. 55, AMS, 2008.

7. F. D'Andrea and L. Dąbrowski, The Standard Model in Noncommutative Geometry and Morita equivalence, J. Noncommut. Geom. 10 (2016) 551-578. 
8. L. Dąbrowski, F. D'Andrea and A Sitarz, The Standard Model in noncommutative geometry: fundamental fermions as internal forms, arXiv:1703.05279

9. T. Krajewski, Classification of Finite Spectral Triples, J. Geom. Phys. 28 (1998) $1-30$.

10. S. Lord, A. Rennie and J.C. Várilly, Riemannian manifolds in noncommutative geometry, J. Geom. Phys. 62 (2012) 1611-1638.

11. M. Paschke and A. Sitarz, Discrete spectral triples and their symmetries, J. Math. Phys. 39 (1996) 6191-6205.

12. R.J. Plymen, Strong Morita equivalence, spinors and symplectic spinors, J. Operator Theory 16 (1986), 305-324.

Ludwik Dąbrowski

Scuola Internazionale Superiore di Studi Avanzati (SISSA)

via Bonomea 265

I-34136 Trieste

e-mail: dabrow@sissa.it 